\title{
Dynamical Behavior of a System of Third-Order Rational Difference Equation
}

\author{
Qianhong Zhang, ${ }^{1}$ Jingzhong Liu, ${ }^{2}$ and Zhenguo Luo ${ }^{3}$ \\ ${ }^{1}$ Guizhou Key Laboratory of Economics System Simulation, Guizhou University of Finance and Economics, \\ Guiyang, Guizhou 550004, China \\ ${ }^{2}$ Department of Mathematics and Physics, Hunan Institute of Technology, Hengyang, Hunan 421002, China \\ ${ }^{3}$ Department of Mathematics, Hengyang Normal University, Hengyang, Hunan 421002, China
}

Correspondence should be addressed to Qianhong Zhang; zqianhong68@163.com

Received 31 December 2014; Accepted 3 March 2015

Academic Editor: Leonid Shaikhet

Copyright (C) 2015 Qianhong Zhang et al. This is an open access article distributed under the Creative Commons Attribution License, which permits unrestricted use, distribution, and reproduction in any medium, provided the original work is properly cited.

This paper deals with the boundedness, persistence, and global asymptotic stability of positive solution for a system of third-order rational difference equations $x_{n+1}=A+x_{n} / y_{n-1} y_{n-2}, y_{n+1}=A+y_{n} / x_{n-1} x_{n-2}, n=0,1, \ldots$, where $A \in(0, \infty), x_{-i} \in(0, \infty)$; $y_{-i} \in(0, \infty), i=0,1,2$. Some examples are given to demonstrate the effectiveness of the results obtained.

\section{Introduction}

It is known that difference equation appears naturally as discrete analogous and as numerical solutions of differential equation and delay differential equation having many applications in economics, biology, computer science, control engineering, and so forth. The study of discrete dynamical systems described by difference equations has now been paid great attention by many mathematical researchers. Particularly, the persistence, boundedness, local asymptotic stability, global character, and the existence of positive periodic solutions can be discussed in many papers.

In 1998, DeVault et al. [1] proved every positive solution of the difference equation:

$$
x_{n+1}=A+\frac{x_{n}}{x_{n-1}}, \quad n=0,1, \ldots,
$$

where $A \in(0, \infty)$ oscillates about the positive equilibrium $c=1+A$ of (1). Moreover every positive solution of (1) is bounded away from zero and infinity. Also the positive equilibrium of (1) is globally asymptotically stable.

In 2003, Abu-Saris and DeVault [2] studied the following recursive difference equation:

$$
x_{n+1}=A+\frac{x_{n}}{x_{n-k}}, \quad n=0,1, \ldots,
$$

where $A \in(1,+\infty), x_{-k}, x_{-k+1}, \ldots, x_{0}$ are positive real numbers. For similar results the reader can refer to [3-9].

Difference equations or discrete dynamical systems are a diverse field which impact almost every branch of pure and applied mathematics. We refer to $[10,11]$ for basic theory of difference equations and rational difference equations. It is very interesting to investigate the qualitative behavior of the discrete dynamical systems of nonlinear difference equations. Recently there has been a lot of work concerning the global asymptotic stability, the periodicity, and the boundedness of nonlinear difference equations. Moreover similar results in [12-17] have been derived for systems of two nonlinear difference equations.

Papaschinopoulos and Schinas [12] investigated the global behavior for a system of the following two nonlinear difference equations:

$$
x_{n+1}=A+\frac{y_{n}}{x_{n-p}}, \quad y_{n+1}=A+\frac{x_{n}}{y_{n-q}}, \quad n=0,1, \ldots
$$

where $A$ is a positive real number, $p, q$ are positive integers, and $x_{-p}, \ldots, x_{0}, x_{-q}, \ldots, x_{0}$ are positive real numbers. 
In 2005, Yang [13] studied the global behavior of the following system:

$$
x_{n}=A+\frac{y_{n-1}}{x_{n-p} y_{n-q}}, \quad y_{n}=A+\frac{x_{n-1}}{x_{n-r} y_{n-s}}, \quad n=1,2, \ldots,
$$

where $p \geq 2, q \geq 2, r \geq 2, s \geq 2, A$ is a positive constant, and initial values $x_{1-\max \{p, r\}}, x_{2-\max \{p, r\}}, \ldots, x_{0}, y_{1-\max \{q, s\}}$, $y_{2-\max \{q, s\}}, \ldots, y_{0}$ are positive real numbers.

In 2012, Zhang et al. [14] investigated the global behavior for a system of the following third-order nonlinear difference equations:

$$
x_{n+1}=\frac{x_{n-2}}{B+y_{n-2} y_{n-1} y_{n}}, \quad y_{n+1}=\frac{y_{n-2}}{A+x_{n-2} x_{n-1} x_{n}}
$$

where $A, B \in(0, \infty)$, and the initial values $x_{-i}, y_{-i} \in(0, \infty)$, $i=0,1,2$.

Motivated by the above mentioned discussion, in this paper we study the behavior of solutions of the following system:

$$
\begin{array}{r}
x_{n+1}=A+\frac{x_{n}}{y_{n-1} y_{n-2}}, \\
y_{n+1}=A+\frac{y_{n}}{x_{n-1} x_{n-2}}, \\
n=0,1, \ldots,
\end{array}
$$

where $A$ is a positive constant. And initial conditions $x_{-i}$, $y_{-i} \in(0, \infty), i=0,1,2$.

Clearly, if $A>0$, system (6) has always positive equilibrium. Consider

$$
(c, c)=\left(\frac{A+\sqrt{A^{2}+4}}{2}, \frac{A+\sqrt{A^{2}+4}}{2}\right) .
$$

If $1<A<2 / \sqrt{3}$, system (6) has two additional positive equilibria:

$$
\begin{aligned}
& \left(a_{1}, b_{1}\right)=\left(\frac{2}{A-\sqrt{4-3 A^{2}}}, \frac{2}{A+\sqrt{4-3 A^{2}}}\right), \\
& \left(a_{2}, b_{2}\right)=\left(\frac{2}{A+\sqrt{4-3 A^{2}}}, \frac{2}{A-\sqrt{4-3 A^{2}}}\right) .
\end{aligned}
$$

Our results in this paper are listed below:

(i) If $A>1$, every positive solution of system (6) is bounded.

(ii) If $A>2 / \sqrt{3},(c, c)$ is locally asymptotically stable.

(iii) If $A>\sqrt{3}$, every positive solution of (6) approaches $(c, c)$.

(iv) If $1<A<2 / \sqrt{3},\left(a_{1}, b_{1}\right)$ and $\left(a_{2}, b_{2}\right)$ are locally asymptotically stable.

\section{Boundedness}

Theorem 1. Let $\left\{\left(x_{n}, y_{n}\right)\right\}$ be a positive solution of (6). Then the following statements hold:

(i) $x_{n} \geq A$ and $y_{n} \geq A$ for all $n \geq 1$.

(ii) If $A>1$, then, for $k \geq 4$, we have the following:

$$
\begin{aligned}
& x_{k} \leq \frac{1}{\left(A^{2}\right)^{k-3}}\left(x_{3}-\frac{A^{3}}{A^{2}-1}\right)+\frac{A^{3}}{A^{2}-1}, \\
& y_{k} \leq \frac{1}{\left(A^{2}\right)^{k-3}}\left(y_{3}-\frac{A^{3}}{A^{2}-1}\right)+\frac{A^{3}}{A^{2}-1} .
\end{aligned}
$$

Proof. Assertion (i) is obviously true. We now prove assertion (ii). From (6) and in view of (i), we have, for all $k \geq 4$, that

$$
\begin{aligned}
& x_{k}=A+\frac{x_{k-1}}{y_{k-2} y_{k-3}} \leq A+\frac{1}{A^{2}} x_{k-1}, \\
& y_{k}=A+\frac{y_{k-1}}{x_{k-2} x_{k-3}} \leq A+\frac{1}{A^{2}} y_{k-1} .
\end{aligned}
$$

Let $v_{k}, w_{k}$ be the solution of following system, respectively,

$$
v_{k}=A+\frac{1}{A^{2}} v_{k-1}, \quad w_{k}=A+\frac{1}{A^{2}} w_{k-1},
$$

such that

$$
x_{3}=v_{3}, \quad y_{3}=w_{3} \text {. }
$$

We prove by induction that

$$
x_{k} \leq v_{k}, \quad y_{k} \leq w_{k}, \quad k \geq 4
$$

Suppose that (13) is true for $k=m \geq 4$. Then from (10) we get the following:

$$
\begin{aligned}
& x_{m+1} \leq A+\frac{1}{A^{2}} x_{m} \leq A+\frac{1}{A^{2}} v_{m}=v_{m+1}, \\
& y_{m+1} \leq A+\frac{1}{A^{2}} y_{m} \leq A+\frac{1}{A^{2}} w_{m}=w_{m+1} .
\end{aligned}
$$

Therefore (13) is true. From (11) we have the following:

$$
\begin{array}{r}
v_{k}=\frac{1}{\left(A^{2}\right)^{k-3}}\left(x_{3}-\frac{A^{3}}{A^{2}-1}\right)+\frac{A^{3}}{A^{2}-1}, \\
w_{k}=\frac{1}{\left(A^{2}\right)^{k-3}}\left(y_{3}-\frac{A^{3}}{A^{2}-1}\right)+\frac{A^{3}}{A^{2}-1}, \\
k \geq 4 .
\end{array}
$$

Then from (10), (13), and (14) the proof of the relation (9) follows immediately. 


\section{Stability}

Theorem 2. Assume that $A>2 / \sqrt{3}$. Then the equilibrium $(c, c)=\left(\left(A+\sqrt{A^{2}+4}\right) / 2,\left(A+\sqrt{A^{2}+4}\right) / 2\right)$ is locally asymptotically stable.

Proof. We can obtain easily that the linearized system of (6) about the positive equilibrium $(c, c)$ is

$$
\Psi_{n+1}=B \Psi_{n}
$$

where

$$
\Psi_{n}=\left(\begin{array}{c}
x_{n} \\
x_{n-1} \\
x_{n-2} \\
y_{n} \\
y_{n-1} \\
y_{n-2}
\end{array}\right)
$$$$
D B D^{-1}=\left(\begin{array}{cccccc}
c^{-2} & 0 & 0 & 0 & -c^{-2} d_{1} d_{5}^{-1} & -c^{-2} d_{1} d_{6}^{-1} \\
d_{2} d_{1}^{-1} & 0 & 0 & 0 & 0 & 0 \\
0 & d_{3} d_{2}^{-1} & 0 & 0 & 0 & 0 \\
0 & -c^{-2} d_{4} d_{2}^{-1} & -c^{-2} d_{4} d_{3}^{-1} & c^{-2} & 0 & 0 \\
0 & 0 & 0 & d_{5} d_{4}^{-1} & 0 & 0 \\
0 & 0 & 0 & 0 & d_{6} d_{5}^{-1} & 0
\end{array}\right)
$$

From $d_{1}>d_{2}>d_{3}>0$ and $d_{4}>d_{5}>d_{6}>0$ imply that

$$
d_{2} d_{1}^{-1}<1, \quad d_{3} d_{2}^{-1}<1, \quad d_{5} d_{4}^{-1}<1, \quad d_{6} d_{5}^{-1}<1 .
$$

Furthermore,

$$
\begin{aligned}
c^{-2}+c^{-2} d_{1} d_{5}^{-1}+c^{-2} d_{1} d_{6}^{-1} & =c^{-2}\left(1+d_{1} d_{5}^{-1}+d_{1} d_{6}^{-1}\right) \\
& =c^{-2}\left(1+\frac{1}{1-5 \varepsilon}+\frac{1}{1-6 \varepsilon}\right) \\
& <c^{-2} \frac{3}{1-6 \varepsilon}<1, \\
c^{-2}+c^{-2} d_{4} d_{2}^{-1}+c^{-2} d_{4} d_{3}^{-1} & =c^{-2}\left(1+d_{4} d_{2}^{-1}+d_{4} d_{3}^{-1}\right) \\
& =c^{-2}\left(1+\frac{1}{1-2 \varepsilon}+\frac{1}{1-3 \varepsilon}\right) \\
& <c^{-2} \frac{3}{1-3 \varepsilon}<c^{-2} \frac{3}{1-6 \varepsilon}<1 .
\end{aligned}
$$

$$
B=\left(\begin{array}{cccccc}
c^{-2} & 0 & 0 & 0 & -c^{-2} & -c^{-2} \\
1 & 0 & 0 & 0 & 0 & 0 \\
0 & 1 & 0 & 0 & 0 & 0 \\
0 & -c^{-2} & -c^{-2} & c^{-2} & 0 & 0 \\
0 & 0 & 0 & 1 & 0 & 0 \\
0 & 0 & 0 & 0 & 1 & 0
\end{array}\right) .
$$

Let $\lambda_{1}, \lambda_{2}, \ldots, \lambda_{6}$ denote the eigenvalues of matrix $B$, let $D=$ $\operatorname{diag}\left(d_{1}, d_{2}, \ldots, d_{6}\right)$ be a diagonal matrix, where $d_{1}=d_{4}=1$, $d_{k}=1-k \varepsilon(k=2,3,5,6)$, and

$$
0<\varepsilon<\min \left\{\frac{1}{6}, \frac{c^{2}-3}{6 c^{2}}\right\}
$$

Clearly, $D$ is invertible. Computing matrix $D B D^{-1}$, we obtain that

It is well known that $B$ has the same eigenvalues as $D B D^{-1}$ : we have that

$$
\begin{aligned}
\max _{1 \leq i \leq 6}\left|\lambda_{i}\right| \leq\left\|D B D^{-1}\right\|_{\infty} & \\
=\max \{ & d_{2} d_{1}^{-1}, d_{3} d_{2}^{-1}, d_{5} d_{4}^{-1}, d_{6} d_{5}^{-1}, \\
& c^{-2}\left(1+d_{1} d_{5}^{-1}+d_{1} d_{6}^{-1}\right) \\
& \left.c^{-2}\left(1+d_{4} d_{2}^{-1}+d_{4} d_{3}^{-1}\right)\right\}<1 .
\end{aligned}
$$

This implies that the equilibrium $(c, c)$ of (6) is locally asymptotically stable.

To examine the global attractivity of $(c, c)$, we need the following result.

Lemma 3. Assume $\alpha>\beta>\sqrt{3}$. Then $\alpha^{3} /\left(\alpha^{2}-1\right)>\beta^{3} /\left(\beta^{2}-\right.$ 1).

Proof. Consider the function $f(x)=x^{3} /\left(x^{2}-1\right)$. For $x>\sqrt{3}$, we have

$$
f^{\prime}(x)=\frac{x^{2}(x+\sqrt{3})(x-\sqrt{3})}{\left(x^{2}-1\right)^{2}}>0 .
$$


So $f(x)$ is strictly increasing for $x>\sqrt{3}$. We derive that $\alpha^{3} /\left(\alpha^{2}-1\right)>\beta^{3} /\left(\beta^{2}-1\right)$.

Theorem 4. Assume that $A>\sqrt{3}$. Then every positive solution of (6) converges to $(c, c)$.

Proof. Let $\left\{x_{n}, y_{n}\right\}$ be an arbitrary positive solution of (6). Let

$$
\begin{aligned}
& \Lambda_{1}=\lim _{n \rightarrow \infty} \sup \left\{x_{n}, x_{n+1}, \ldots\right\}, \\
& \lambda_{1}=\lim _{n \rightarrow \infty} \inf \left\{x_{n}, x_{n+1}, \ldots\right\}, \\
& \Lambda_{2}=\lim _{n \rightarrow \infty} \sup \left\{y_{n}, y_{n+1}, \ldots\right\}, \\
& \lambda_{1}=\lim _{n \rightarrow \infty} \inf \left\{y_{n}, y_{n+1}, \ldots\right\} .
\end{aligned}
$$

From Theorem 1, we have $0<A \leq \lambda_{1} \leq \Lambda_{1}<\infty, 0<A \leq$ $\lambda_{2} \leq \Lambda_{2}<\infty$. The previous and (6) imply that

$$
\begin{array}{ll}
\Lambda_{1} \leq A+\frac{\Lambda_{1}}{\lambda_{2}^{2}}, & \Lambda_{2} \leq A+\frac{\Lambda_{2}}{\lambda_{1}^{2}}, \\
\lambda_{1} \geq A+\frac{\lambda_{1}}{\Lambda_{2}^{2}}, & \lambda_{2} \geq A+\frac{\lambda_{2}}{\Lambda_{1}^{2}},
\end{array}
$$

which can derive that

$$
\begin{aligned}
& \Lambda_{1} \Lambda_{2}^{2}\left(\lambda_{2}^{2}-1\right) \leq \lambda_{1} \lambda_{2}^{2}\left(\Lambda_{2}^{2}-1\right) \\
& \Lambda_{2} \Lambda_{1}^{2}\left(\lambda_{1}^{2}-1\right) \leq \lambda_{2} \lambda_{1}^{2}\left(\Lambda_{1}^{2}-1\right) .
\end{aligned}
$$

It follows that $\Lambda_{1}^{3} \Lambda_{2}^{3}\left(\lambda_{1}^{2}-1\right)\left(\lambda_{2}^{2}-1\right) \leq \lambda_{1}^{3} \lambda_{2}^{3}\left(\Lambda_{1}^{2}-1\right)\left(\Lambda_{2}^{2}-1\right)$; namely,

$$
\frac{\Lambda_{1}^{3} \Lambda_{2}^{3}}{\left(\Lambda_{1}^{2}-1\right)\left(\Lambda_{2}^{2}-1\right)} \leq \frac{\lambda_{1}^{3} \lambda_{2}^{3}}{\left(\lambda_{1}^{2}-1\right)\left(\lambda_{2}^{2}-1\right)}
$$

If $\Lambda_{1}>\lambda_{1}$ and $\Lambda_{2}>\lambda_{2}$, it follows from Lemma 3 and the condition $A>\sqrt{3}$ that $\Lambda_{1}^{3} \Lambda_{2}^{3} /\left(\Lambda_{1}^{2}-1\right)\left(\Lambda_{2}^{2}-1\right)>\lambda_{1}^{3} \lambda_{2}^{3} /\left(\lambda_{1}^{2}-\right.$ $1)\left(\lambda_{2}^{2}-1\right)$. Then a contradiction occurs. Thus we have either $\Lambda_{1}=\lambda_{1}$ or $\Lambda_{2}=\lambda_{2}$.

We assume $\Lambda_{1}=\lambda_{1}$ (the discussion for the case $\Lambda_{2}=\lambda_{2}$ is similar). Then $\lim _{n \rightarrow \infty} x_{n}$ exists. Using (27) and in view of $\Lambda_{1}=\lambda_{1}$, it is obvious that $\Lambda_{2} \leq \lambda_{2}$, so $\Lambda_{2}=\lambda_{2}$. Then $\lim _{n \rightarrow \infty} y_{n}$ exists. From the uniqueness of the positive equilibrium $(c, c)$ of (6), we conclude that $\lim _{n \rightarrow \infty} x_{n}=c$, $\lim _{n \rightarrow \infty} y_{n}=c$.

Combining Theorems 2 and 4, we obtain the following theorem.

Theorem 5. Assume that $A>\sqrt{3}$. Then the positive equilibrium $(c, c)$ of $(6)$ is globally asymptotically stable for all positive solutions.

Theorem 6. If $1<A<2 / \sqrt{3}$, then the equilibria $\left(a_{1}, b_{1}\right)$ and $\left(a_{2}, b_{2}\right)$ are locally asymptotically stable.

Proof. The proof is similar to the proof of Theorem 2. We can obtain easily that the linearized system of (6) about the positive equilibrium $\left(a_{1}, b_{1}\right)$ is

$$
\Psi_{n+1}=H \Psi_{n}
$$

where

$$
H=\left(\begin{array}{c}
x_{n} \\
x_{n-1} \\
x_{n-2} \\
y_{n} \\
y_{n-1} \\
y_{n-2}
\end{array}\right),
$$

Let $\lambda_{1}^{\prime}, \lambda_{2}^{\prime}, \ldots, \lambda_{6}^{\prime}$ denote the eigenvalues of matrix $H$, let $D=$ $\operatorname{diag}\left(d_{1}, d_{2}, \ldots, d_{6}\right)$ be a diagonal matrix, where $d_{1}=d_{4}=1$, $d_{k}=1-k \varepsilon(k=2,3,5,6)$, and

$$
0<\varepsilon^{\prime}<\min \left\{\frac{1}{6}, \frac{a_{1}^{2}-3}{6 a_{1}^{2}}, \frac{b_{1}^{3}-3 a_{1}}{6 b_{1}^{2}}\right\} .
$$

Clearly, $H$ is invertible. Computing matrix $D H D^{-1}$, we obtain that

$$
D H D^{-1}=\left(\begin{array}{cccccc}
b_{1}^{-2} & 0 & 0 & 0 & -a_{1} b_{1}^{-3} d_{1} d_{5}^{-1} & -a_{1} b_{1}^{-3} d_{1} d_{6}^{-1} \\
d_{2} d_{1}^{-1} & 0 & 0 & 0 & 0 & 0 \\
0 & d_{3} d_{2}^{-1} & 0 & 0 & 0 & 0 \\
0 & -b_{1} a_{1}^{-3} d_{4} d_{2}^{-1} & -b_{1} a_{1}^{-3} d_{4} d_{3}^{-1} & a_{1}^{-2} & 0 & 0 \\
0 & 0 & 0 & d_{5} d_{4}^{-1} & 0 & 0 \\
0 & 0 & 0 & 0 & d_{6} d_{5}^{-1} & 0
\end{array}\right)
$$


From $d_{1}>d_{2}>d_{3}>0$ and $d_{4}>d_{5}>d_{6}>0$ we imply that

$$
d_{2} d_{1}^{-1}<1, \quad d_{3} d_{2}^{-1}<1, \quad d_{5} d_{4}^{-1}<1, \quad d_{6} d_{5}^{-1}<1 .
$$

Furthermore,

$$
\begin{aligned}
b_{1}^{-2} & +a_{1} b_{1}^{-3} d_{1} d_{5}^{-1}+a_{1} b_{1}^{-3} d_{1} d_{6}^{-1} \\
& =b_{1}^{-2}\left(1+\frac{a_{1}}{b_{1}} d_{1} d_{5}^{-1}+\frac{a_{1}}{b_{1}} d_{1} d_{6}^{-1}\right) \\
& <\frac{a_{1}}{b_{1}^{3}}\left(1+\frac{1}{1-5 \varepsilon^{\prime}}+\frac{1}{1-6 \varepsilon^{\prime}}\right)<\frac{a_{1}}{b_{1}^{3}} \frac{3}{1-6 \varepsilon^{\prime}}<1, \\
a_{1}^{-2} & +b_{1} a_{1}^{-3} d_{4} d_{2}^{-1}+b_{1} a_{1}^{-3} d_{4} d_{3}^{-1} \\
& =a_{1}^{-2}\left(1+\frac{b_{1}}{a_{1}} d_{4} d_{2}^{-1}+\frac{b_{1}}{a_{1}} d_{4} d_{3}^{-1}\right) \\
& <\frac{1}{a_{1}^{2}}\left(1+\frac{1}{1-2 \varepsilon^{\prime}}+\frac{1}{1-3 \varepsilon^{\prime}}\right)<\frac{1}{a_{1}^{2}} \frac{3}{1-6 \varepsilon^{\prime}}<1 .
\end{aligned}
$$

It is well known that $H$ has the same eigenvalues as $D H D^{-1}$; we have that

$$
\begin{aligned}
\max _{1 \leq i \leq 6}\left|\lambda_{i}^{\prime}\right| \leq\left\|D H D^{-1}\right\|_{\infty} & \\
= & \max \left\{d_{2} d_{1}^{-1}, d_{3} d_{2}^{-1}, d_{5} d_{4}^{-1}, d_{6} d_{5}^{-1},\right. \\
& b_{1}^{-2}+a_{1} b_{1}^{-3} d_{1} d_{5}^{-1}+a_{1} b_{1}^{-3} d_{1} d_{6}^{-1}, \\
& \left.a_{1}^{-2}+b_{1} a_{1}^{-3} d_{4} d_{2}^{-1}+b_{1} a_{1}^{-3} d_{4} d_{3}^{-1}\right\}<1 .
\end{aligned}
$$

This implies that the equilibrium $\left(a_{1}, b_{1}\right)$ of $(6)$ is locally asymptotically stable.

Similarly, we can prove that the equilibrium $\left(a_{2}, b_{2}\right)$ is locally asymptotically stable.

\section{Numerical Example}

In order to illustrate the results of the previous sections and to support our theoretical discussions, we consider several interesting numerical examples in this section. These examples represent different types of qualitative behavior of solutions to nonlinear difference equations and system of nonlinear difference equations.

Example 1. Consider the following system of difference equations:

$$
x_{n+1}=A+\frac{x_{n}}{y_{n-1} y_{n-2}}, \quad y_{n+1}=A+\frac{y_{n}}{x_{n-1} x_{n-2}},
$$

with $A=2.4>2 / \sqrt{3}$ and the initial values $x_{-i}=$ $1.2, y_{-i}=0.5(i=0,1,2)$. Then the solution $\left(x_{n}, y_{n}\right)$ of system (35) is bounded and persists. And the system has a unique equilibrium $(x, y)=(2.7620,2.7620)$, which is globally asymptotically stable (see Figure 1).

Let $A=1.1$ and the initial values $x_{-i}=2, y_{-i}=1.2(i=$ $0,1,2)$. Then the solution $\left(x_{n}, y_{n}\right)$ of system (35) is bounded and persists (see Figure 2).

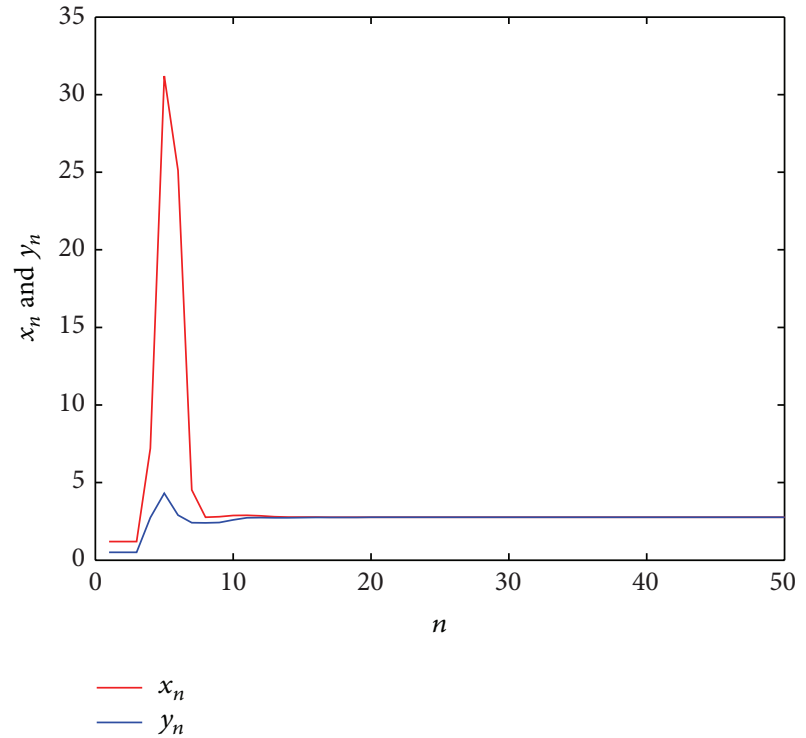

Figure 1: The dynamics of system (35) at $A=2.4$.

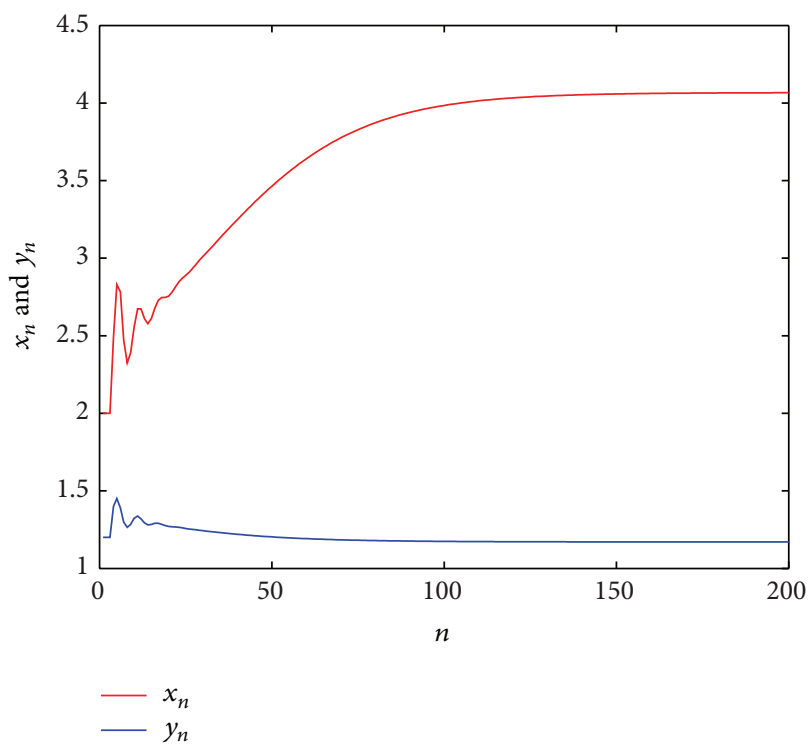

Figure 2: The dynamics of system (35) at $A=1.1$.

\section{Conflict of Interests}

The authors declare that there is no conflict of interests regarding the publication of this paper.

\section{Acknowledgments}

This work was financially supported by the National Natural Science Foundation of China (Grant no. 11361012), the China Postdoctoral Science Foundation (no. 2013T60934), and the Scientific Research Foundation of Guizhou Provincial Science and Technology Department ([2013]J2083). 


\section{References}

[1] R. DeVault, G. Ladas, and S. W. Schultz, "On the recursive sequence $x_{n+1=} A / x_{n}+1 / x_{n-2}$," Proceedings of the American Mathematical Society, vol. 126, no. 11, pp. 3257-3261, 1998.

[2] R. M. Abu-Saris and R. DeVault, "Global stability of $y_{n+1}=A+$ $\frac{y_{n}}{y_{n-k}}$, , Applied Mathematics Letters, vol. 16, no. 2, pp. 173-178, 2003.

[3] A. M. Amleh, E. A. Grove, G. Ladas, and D. A. Georgiou, "On the recursive sequence $x_{n+1}=A+x_{n-1} / x_{n}$ " Journal of Mathematical Analysis and Applications, vol. 233, no. 2, pp. 790$798,1999$.

[4] W. S. He, W. T. Li, and X. X. Yan, "Global attractivity of the difference equation $x_{n+1}=a+x_{n-k} / x_{n}$," Applied Mathematics and Computation, vol. 151, pp. 879-885, 2004.

[5] R. DeVault, G. Ladas, and S. W. Schultz, "Necessary and sufficient conditions for the boundedness of $x_{n+1}=A / x_{n}^{p}+$ $B / x_{n-1}^{q}$, Journal of Difference Equations and Applications, vol. 3, no. 3-4, pp. 259-266, 1998.

[6] R. P. Agarwal, W.-T. Li, and P. Y. H. Pang, "Asymptotic behavior of a class of nonlinear delay difference equations," Journal of Difference Equations and Applications, vol. 8, no. 8, pp. 719-728, 2002.

[7] W.-T. Li and H.-R. Sun, "Dynamics of a rational difference equation," Applied Mathematics and Computation, vol. 163, no. 2, pp. 577-591, 2005.

[8] Y.-H. Su and W.-T. Li, "Global attractivity of a higher order nonlinear difference equation," Journal of Difference Equations and Applications, vol. 11, no. 10, pp. 947-958, 2005.

[9] L.-X. Hu and W.-T. Li, "Global stability of a rational difference equation," Applied Mathematics and Computation, vol. 190, no. 2, pp. 1322-1327, 2007.

[10] V. L. Kocic and G. Ladas, Global Behavior of Nonlinear Difference Equations of Higher Order with Application, Kluwer Academic Publishers, Dordrecht, The Netherlands, 1993.

[11] M. R. C. Kulenonvic and G. Ladas, Dynamics of Second Order Rational Difference Equations with Open Problems and Conjectures, Chapman \& Hall, CRC Press, Boca Raton, Fla, USA, 2002.

[12] G. Papaschinopoulos and C. J. Schinas, "On a system of two nonlinear difference equations," Journal of Mathematical Analysis and Applications, vol. 219, no. 2, pp. 415-426, 1998.

[13] X. Yang, "On the system of rational difference equations $x_{n}=$ $A+\left(y_{n-1} / x_{n-p} y_{n-q}\right), y_{n}=A+x_{n-1} / x_{n-r} y_{n-s}$," Journal of Mathematical Analysis and Applications, vol. 307, pp. 305-311, 2005.

[14] Q. Zhang, L. Yang, and J. Liu, "Dynamics of a system of rational third-order difference equation," Advances in Difference Equations, vol. 2012, article 136, pp. 1-6, 2012.

[15] Q. Zhang and W. Zhang, "On a system of two high-order nonlinear difference equations," Advances in Mathematical Physics, vol. 2014, Article ID 729273, 8 pages, 2014.

[16] Q. Din, T. F. Ibrahim, and K. A. Khan, "Behavior of a competitive system of second-order difference equations," The Scientific World Journal, vol. 2014, Article ID 283982, 9 pages, 2014.

[17] Q. Din, K. A. Khan, and A. Nosheen, "Stability analysis of a system of exponential difference equations," Discrete Dynamics in Nature and Society, vol. 2014, Article ID 375890, 11 pages, 2014. 


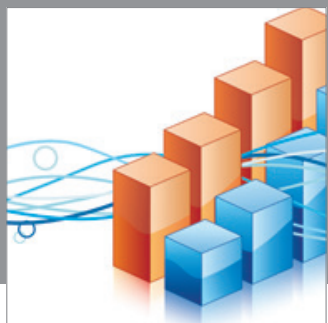

Advances in

Operations Research

mansans

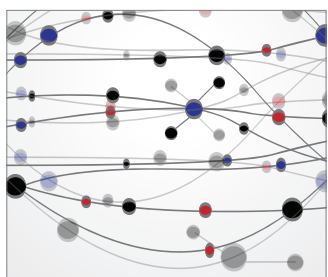

The Scientific World Journal
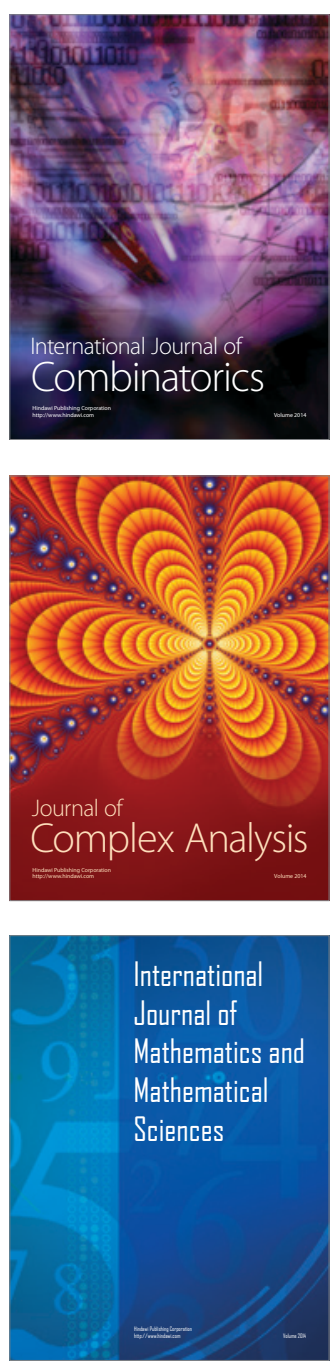
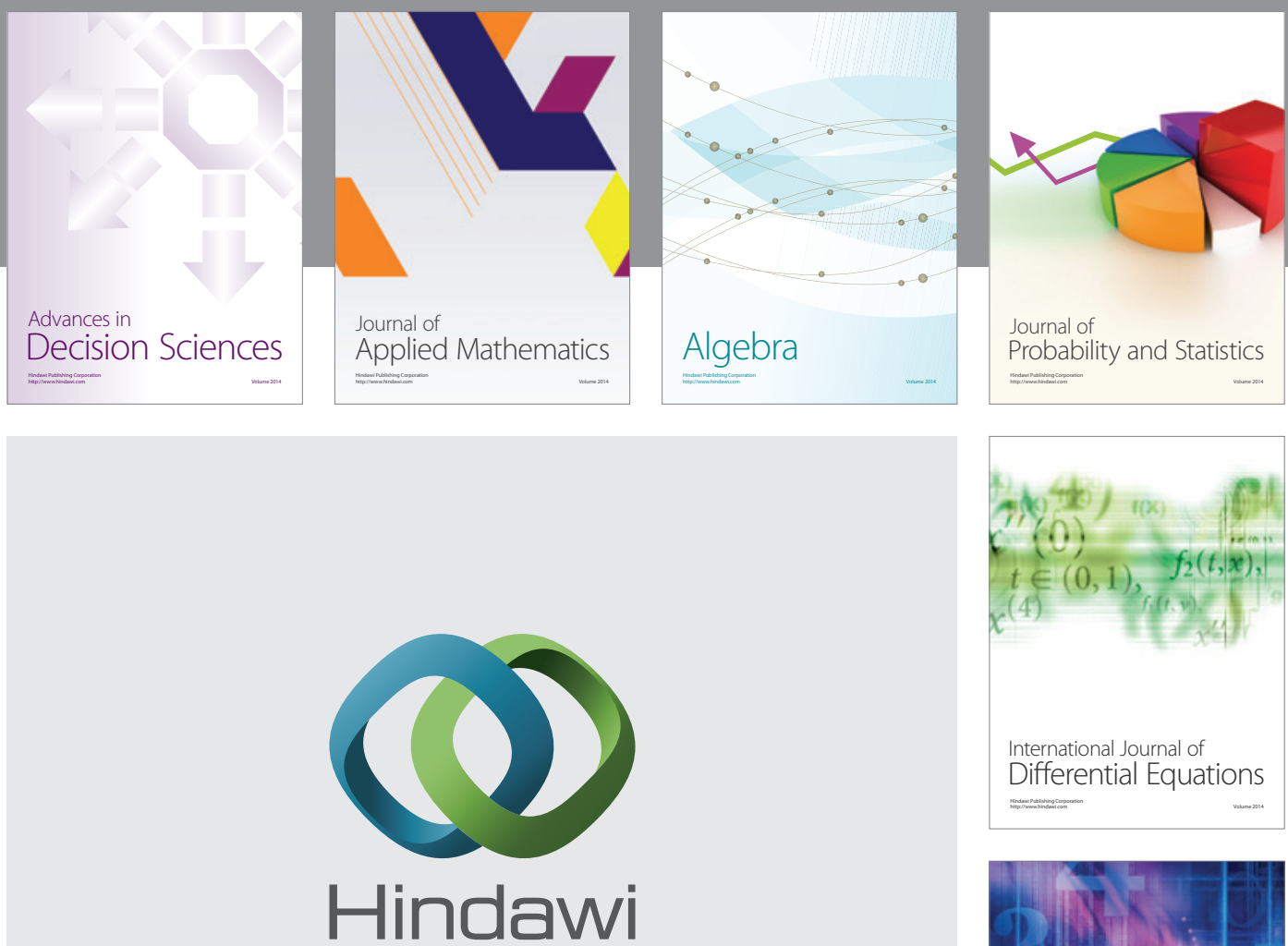

Submit your manuscripts at http://www.hindawi.com
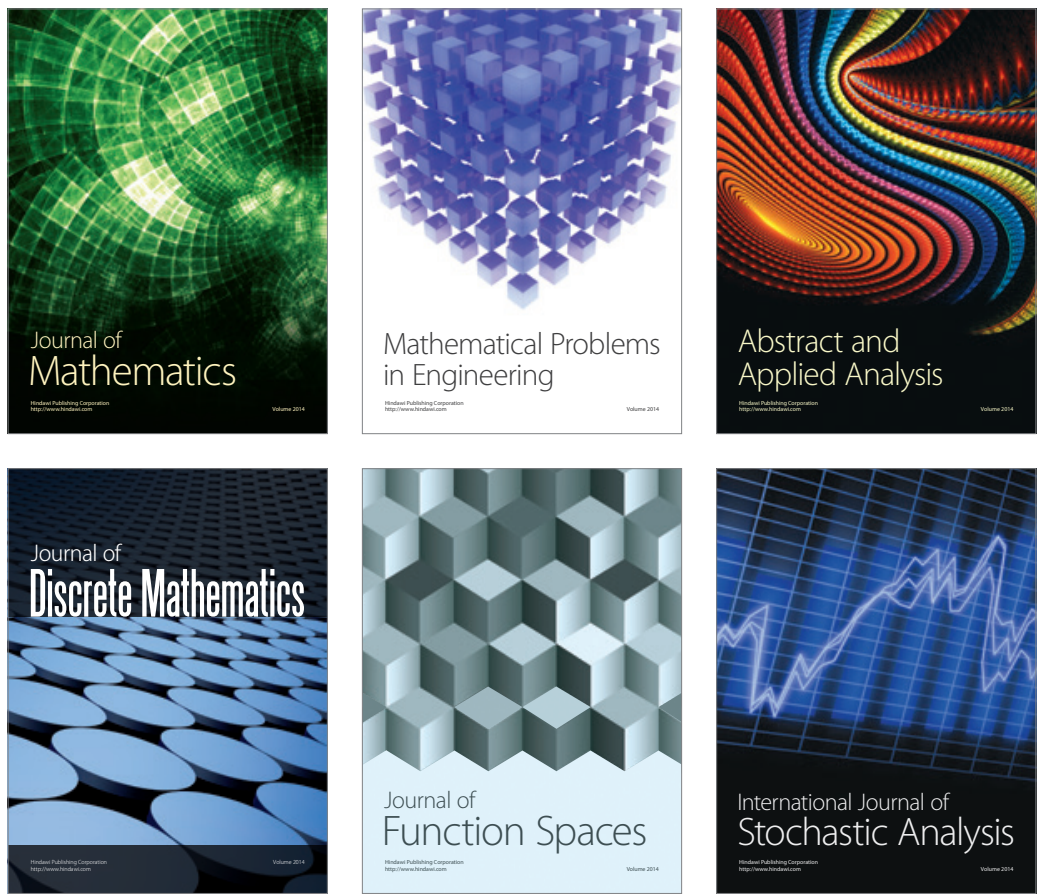

Journal of

Function Spaces

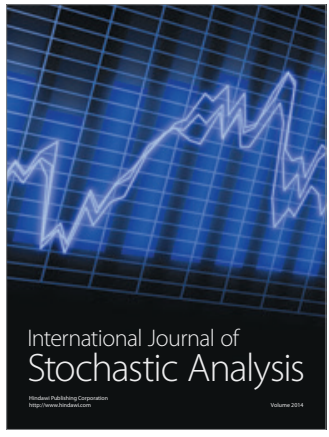

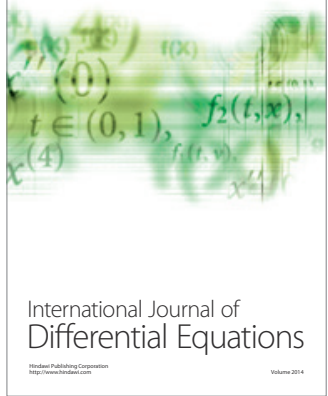
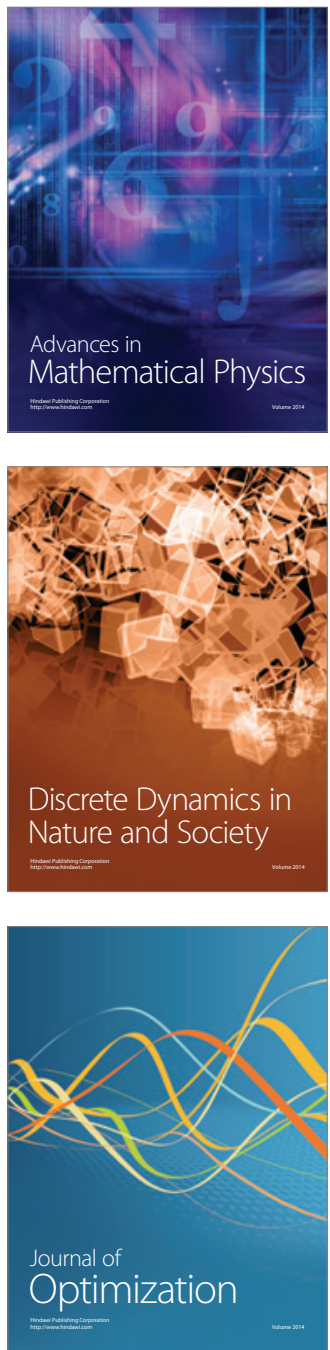